\title{
NONLOCAL PROBLEMS AT NEARLY CRITICAL GROWTH
}

\author{
SUNRA MOSCONI AND MARCO SQUASSINA
}

\begin{abstract}
We study the asymptotic behavior of solutions to the nonlocal nonlinear equation $\left(-\Delta_{p}\right)^{s} u=|u|^{q-2} u$ in a bounded domain $\Omega \subset \mathbb{R}^{N}$ as $q$ approaches the critical Sobolev exponent $p^{*}=N p /(N-p s)$. We prove that ground state solutions concentrate at a single point $\bar{x} \in \bar{\Omega}$ and analyze the asymptotic behavior for sequences of solutions at higher energy levels. In the semi-linear case $p=2$, we prove that for smooth domains the concentration point $\bar{x}$ cannot lie on the boundary, and identify its location in the case of annular domains.
\end{abstract}

\section{INTRODUCTION AND MAIN RESULTS}

Let $\Omega$ be a smooth bounded domain of $\mathbb{R}^{N}, N>p s, s \in(0,1)$ and $p>1$. For any sufficiently small $\varepsilon>0$, we consider the nonlocal nonlinear problem

$$
\begin{cases}\left(-\Delta_{p}\right)^{s} u=|u|^{p^{*}-2-\varepsilon} u, & \text { in } \Omega, \\ u=0 & \text { in } \mathbb{R}^{N} \backslash \Omega,\end{cases}
$$

where $p^{*}=N p /(N-s p)$ is the critical exponent for the immersion of

$$
W_{0}^{s, p}(\Omega):=\left\{u \in L^{p}\left(\mathbb{R}^{N}\right): \int_{\mathbb{R}^{2 N}} \frac{|u(x)-u(y)|^{p}}{|x-y|^{N+p s}} d x<+\infty, \quad u=0 \text { in } \mathbb{R}^{N} \backslash \Omega\right\}
$$

into the space $L^{q}(\Omega)$. By a weak solution to problem (1.1) we mean a critical point for the $C^{1}$ functional $I_{\varepsilon}: W_{0}^{s, p}(\Omega) \rightarrow \mathbb{R}$ defined by

$$
I_{\varepsilon}(u)=\frac{1}{p} \int_{\mathbb{R}^{2 N}} \frac{|u(x)-u(y)|^{p}}{|x-y|^{N+s p}} d x d y-\frac{1}{p^{*}-\varepsilon} \int_{\Omega}|u|^{p^{*}-\varepsilon} d x .
$$

The nonlinear operator $\left(-\Delta_{p}\right)^{s}: W_{0}^{s, p}(\Omega) \rightarrow W^{-s, p}(\Omega)$ is defined (up to a multiplicative constant which we will ignore in the following) as the differential of the first term in $I_{\varepsilon}$ and it can be represented, on smooth functions, by

$$
\left(-\Delta_{p}\right)^{s} u(x)=2 \lim _{\varepsilon \searrow 0} \int_{\mathbb{R}^{N} \backslash B_{\varepsilon}(x)} \frac{|u(x)-u(y)|^{p-2}(u(x)-u(y))}{|x-y|^{N+s p}} d y, \quad x \in \mathbb{R}^{N} .
$$

Solutions of (1.1) inherit some mild smoothness when seen as general non-homogeneous nonlocal equations. The regularity theory for $p \neq 2$ is far from complete, however the basic continuity instances of it are covered in [16], [5] at the interior and in [14] at the boundary. In this paper, we are interested in the asymptotic behavior of a sequence of solutions $\left\{u_{\varepsilon}\right\}_{\varepsilon>0}$ to $(1.1)$ as $\varepsilon \searrow 0$, as determined by the limit energy $c=\lim _{\varepsilon \rightarrow 0} I_{\varepsilon}\left(u_{\varepsilon}\right)$.

The interest in such "nearly critical" problems arises from the fact that for $\varepsilon>0$ compactness is recovered and the problem is more easily solved, hopefully providing in the limit a solution to the non-compact problem at $\varepsilon=0$. In many cases, however, the validity of a

2010 Mathematics Subject Classification. 34K37, 58K05.

Key words and phrases. Nonlinear nonlocal equation, critical embedding, nearly critical nonlinearities.

The authors were partially supported by Gruppo Nazionale per l'Analisi Matematica, la Probabilità e le loro Applicazioni (INdAM). 
Pohozaev identity rules out existence of nontrivial solutions for $\varepsilon=0$, and the asymptotic behavior of the approximating solutions describes the phenomenon of lack of solutions in the limit.

In the seminal paper [1], the asymptotic behaviour of the (unique and radial) solution $u_{\varepsilon}$

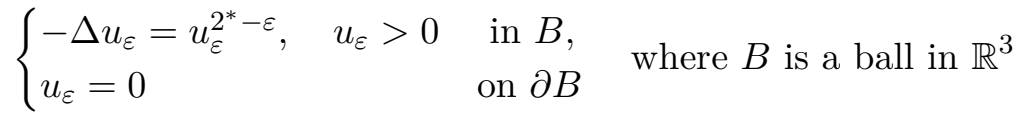

is considered, showing, among other things, that $u_{\varepsilon}$ concentrates at a single point, the center of $B$, at a rate $\max u_{\varepsilon}=u_{\varepsilon}(0) \simeq \sqrt{\varepsilon}$. This kind of results were extended and refined in [7]. For general smooth domains, where uniqueness of solutions (and nonexistence of the latter for $\varepsilon=0)$ to (1.2) is lost, the same kind of behavior is proved in [12], [23] for the ground states of (1.2), namely, nontrivial solutions minimizing the associated energy functional. Indeed, regardless of the existence of positive solutions of the limiting equation, ground states always concentrate all their mass at some point, which is therefore called the point of concentration. Through a rather fine analysis, the concentration point is shown to be a critical point of the Robin function of $\Omega$. For smooth domains, this implies that the concentration points cannot belong to $\partial \Omega$, while for nonsmooth domain the boundary concentration phenomenon can happen, as shown e.g. in [9].

For more general, nonlinear equations, the situation is less clear. In [11] the concentration of ground states is proved for the $p$-Laplacian via critical point methods, while in [20] via $\Gamma$-convergence ones (see the latter for more references on this approach). In [18] more general and non regular operators are considered. However, the location of concentration points for ground states is not clear, (even trying to prove that they do not belong to $\partial \Omega$ in smooth domains), and precise asymptotic behavior of the maxima are even less so. It is worth noting, however, that for a different but related problem involving the $p$-Laplacian, the location of concentration points has been determined with the technique of $p$-harmonic transplantation, see [8].

Regarding the nonlocal problem (1.1), the semi-linear case $p=2$ is considered in [22] with a $\Gamma$-convergence approach and in [21] via profile decomposition. The latter approach relies on the Hilbert structure to take advantage of abstract profile decomposition theory, but, as shown in [15], no such precise decomposition can hold for general bounded sequences when $p \neq 2$. A more suitable profile decomposition when $p \neq 2$ has recently been obtained for Palais-Smale sequences in [6], which in principle may lead to the same kind of results we will discuss in a short while. However, a direct approach through non local Concentration-Compactness seems more convenient for ground state solutions, and is flexible enough to provide informations at higher critical levels as well.

In order to state our main results, let us set

$$
\left|D^{s} u\right|^{p}(x):=\int_{\mathbb{R}^{N}} \frac{|u(x)-u(y)|^{p}}{|x-y|^{N+p s}} d y, \quad \text { for a.e. } x \in \mathbb{R}^{N} .
$$

For general $p>1, s \in] 0,1[$ and $N>p s$ we will prove the following.

Theorem 1.1 (Ground states). Let $\Omega$ be a bounded domain and $\left\{u_{n}\right\}$ be a sequence of ground state solutions to (1.1) for $\varepsilon_{n} \downarrow 0$. For each subsequence (not relabelled) such that $\left|u_{n}\right|^{p^{*}}$ weakly converges in the sense of measure, it holds

(1) $u_{n} \rightarrow 0$ in $L^{q}\left(\mathbb{R}^{N}\right)$ for any $q \in\left[1, p^{*}\right)$;

(2) there exists $\bar{x} \in \bar{\Omega}$ such that $\left|D^{s} u_{n}\right|^{p} \rightarrow \mathbb{S}^{\frac{N}{p s}} \delta_{\bar{x}}$ and $\left|u_{n}\right|^{p^{*}} \rightarrow \mathbb{S}^{\frac{N}{p s}} \delta_{\bar{x}}$ weakly in the sense of measures. 
Theorem 1.2 (Higher states). Let $\Omega, u_{n}, \varepsilon_{n}$ be as above and suppose that

$$
\left.I_{\varepsilon_{n}}\left(u_{n}\right) \rightarrow c \in\right] \frac{s}{N} \mathbb{S}^{\frac{N}{p s}}, 2 \frac{s}{N} \mathbb{S}^{\frac{N}{p s}}[,
$$

and let, up to subsequences, $u_{n} \rightarrow u$ in $W_{0}^{s, p}(\Omega)$. Then one of the following alternatives holds:

(1) $u_{n} \rightarrow u$ strongly in $W_{0}^{s, p}(\Omega)$ with $u$ being a positive or negative solution of the limit equation (1.1) for $\varepsilon=0$.

(2) $u=0$ and there exists $\bar{x} \in \bar{\Omega}$ such that $\left|D^{s} u_{n}\right|^{p} \rightarrow c \frac{N}{s} \delta_{\bar{x}}$ weakly in the sense of measures.

In the case $p=2$ and $\Omega$ smooth we can exclude that the concentration points lie on the boundary, and in some cases precisely locate them.

Theorem 1.3 (Inner concentration). Let $N>2 s$ and $\Omega$ be a bounded $C^{1,1}$ domain. For any $\left\{\varepsilon_{n}\right\}$ with $\varepsilon_{n} \downarrow 0$, let $u_{n}$ be a ground state solution of

$$
\begin{cases}(-\Delta)^{s} u_{n}=u_{n}^{2^{*}-\varepsilon_{n}-1}, & u>0 \text { in } \Omega, \\ u_{n}=0 & \text { in } \Omega^{c} .\end{cases}
$$

Then, up to subsequence, $\left|u_{n}\right|^{2^{*}} \rightarrow \mathbb{S}^{\frac{N}{2 s}} \delta_{\bar{x}}$ weakly in the sense of measures, for some $\bar{x} \in \Omega$.

Theorem 1.4 (Location for annuli). Let $r_{2}>r_{1}>0, \Omega=B_{r_{2}} \backslash B_{r_{1}}$ and $N>2 s$. Then the ground state solutions of (1.4) concentrate at points $\bar{x}$ with $|\bar{x}|$ the harmonic mean of $r_{2}$ and $r_{1}$.

Let us point out the main features of the previous theorems. In order to prove Theorem 1.1, we will derive a Concentration-Compactness alternative for nonlocal problems. In order to do so we proceed directly, controlling through a domain decomposition and Lemma 2.6 below all the nonlocal interactions which, in principle, could contribute to the limiting measures. In a similar way we prove a bound from below on the singular part arising from concentration. One fundamental difficulty in the case $p \neq 2$ is that entire ground state solutions of the limiting problem are unknown explicitly. Thus, we will have to use an auxiliary function recently constructed in [19] to prove that the energy of the ground states converges to the minimal one. To prove Theorem 1.2 we will bound from below the absolutely continuous part of the limiting measures, again through the crucial Lemma 2.6.

For Theorems 1.3 and 1.4 we will employ the Moving Plane method which has recently been proved in [2] in the nonlocal case, together with suitable fractional Kelvin transforms. Our main lemma here is a Harnack inequality on segments for positive solutions $u$ of a large class of semilinear equations. The inequality is of the form

$$
\sup _{[a, b]} u \leq C u(b)
$$

where $[a, b] \subseteq \Omega$ is a segment of suitable fixed length, normal to $\partial \Omega$ at the point $a$, with $C$ being a geometric constant depending only on the domain. For a precise statement see Theorem 4.1. This forces the concentration to happen at least a distance $|b-a|$ away from the boundary. The construction can be performed explicitly for annular domains, yielding Theorem 1.4.

Structure of the paper. In Section 2.1 we fix some notations. In Section 2.2, after recalling some well known facts on the space $W_{0}^{s, p}(\Omega)$ and the ground states solutions of (1.1), we will prove some general results on the weak solutions of (1.1) for fixed $\varepsilon>0$. Section 2.3 is 
dedicated to the nonlocal Concentration-Compactness lemma Theorem 2.5. Then, in Section 3, we will prove Theorems 1.1 and 1.2, and in Section 4 Theorem 1.3 and 1.4.

\section{Preliminaries}

2.1. Notation. For $s \in(0,1), p>1$ and $N>p s$, we let $p^{*}=N p /(N-p s)$. We denote by $\omega_{N}$ the measure of the $N$-dimensional ball having unit radius. For $E \subseteq \mathbb{R}^{N}$ measurable we denote by $|E|$ its $N$-dimensional Lebesgue measure, by $E^{c}=\mathbb{R}^{N} \backslash E$ its complement and by $\chi_{E}$ its characteristic function. If $u: E \rightarrow \mathbb{R}$ is measurable we set

$$
[u]_{W^{s, p}(E)}^{p}:=\int_{E \times E} \frac{|u(x)-u(y)|^{p}}{|x-y|^{N+p s}} d x d y, \quad[u]_{s, p}:=[u]_{W^{s, p}\left(\mathbb{R}^{N}\right)},
$$

and for any $q \geq 1$

$$
|u|_{L^{q}(E)}:=\left(\int_{E}|u|^{q} d x\right)^{1 / q}, \quad|u|_{q}:=|u|_{L^{q}\left(\mathbb{R}^{N}\right)} .
$$

Finally, for $t \in \mathbb{R}$ and $p>1$, we will use the notation

$$
t^{p-1}:=|t|^{p-2} t
$$

For any $u \in W_{0}^{s, p}(\Omega)$ and any Lebesgue point $x$ for $u$, we set

$$
\left|D^{s} u\right|^{p}(x)=\int_{\mathbb{R}^{N}} \frac{|u(x)-u(y)|^{p}}{|x-y|^{N+p s}} d y .
$$

Notice that the following approximate Leibniz formula holds true (2.1) $\forall \theta>0 \exists C_{\theta}$ with

$$
\int_{\mathbb{R}^{N}}\left|D^{s}(u v)\right|^{p} d x \leq(1+\theta) \int_{\mathbb{R}^{N}}\left|D^{s} u\right|^{p}|v|^{p} d x+C_{\theta} \int_{\mathbb{R}^{N}}\left|D^{s} v\right|^{p}|u|^{p} d x
$$

which follows from the elementary inequality $|a+b|^{p} \leq(1+\theta)|a|^{p}+C_{\theta}|b|^{p}$.

2.2. Functional Analytic framework. We let, for any $\Omega \subseteq \mathbb{R}^{N}$,

$$
W_{0}^{s, p}(\Omega):=\left\{u \in L^{p^{*}}\left(\mathbb{R}^{N}\right): u=0 \text { in } \Omega^{c},[u]_{s, p}<+\infty\right\},
$$

which is a Banach space with respect to the norm $[\cdot]_{s, p}$. This space is often denoted by $D_{0}^{s, p}(\Omega)$ in the current literature and it is consistent with the one defined in the introduction as soon as $\Omega$ is bounded. For $N>s p$, the Sobolev inequality reads as

$$
\mathbb{S}:=\inf \left\{\frac{[u]_{s, p}^{p}}{|u|_{p^{*}}^{p}}: u \in W_{0}^{s, p}(\Omega) \backslash\{0\}\right\} .
$$

We recall the following

Proposition 2.1 (Hardy's inequality). Let $N>s p$. Then there exists $C=C(N, p, s)>0$ such that

$$
\int_{\mathbb{R}^{N}} \frac{|u|^{p}}{|x|^{s p}} d x \leq C[u]_{s, p}^{p}, \quad \text { for every } u \in W_{0}^{s, p}\left(\mathbb{R}^{N}\right) .
$$

We will also let, for $1 \leq q \leq p^{*}$,

$$
\mathbb{S}_{q}(\Omega):=\inf \left\{\frac{[u]_{s, p}^{p}}{|u|_{q}^{p}}: u \in W_{0}^{s, p}(\Omega) \backslash\{0\}\right\},
$$

so that $\mathbb{S}=\mathbb{S}_{p^{*}}(\Omega)$. It is a classical fact that $\mathbb{S}_{p^{*}}(\Omega)=\mathbb{S}_{p^{*}}\left(\mathbb{R}^{N}\right)$ for every domain $\Omega$ and that the minimization problem (2.2) for $q=p^{*}$ admits no solution, unless $\Omega=\mathbb{R}^{N}$. For $1<q<p^{*}$ and $\Omega$ bounded, Hölder's inequality ensures that $\mathbb{S}_{q}(\Omega)>0$; moreover, any 
minimizer $u$ for the minimization problem (2.2) (which, actually, exists due to the compactness of $\left.W_{0}^{s, p}(\Omega) \hookrightarrow L^{q}(\Omega)\right)$ is, up to a multiplicative constant, a weak solution of the problem

$$
\begin{cases}\left(-\Delta_{p}\right)^{s} u=|u|^{q-2} u & \text { in } \Omega, \\ u=0 & \text { in } \Omega^{c} .\end{cases}
$$

Clearly any such solution is also a critical point for the functional

$$
J_{q}(u):=\frac{1}{p}[u]_{s, p}^{p}-\frac{1}{q}|u|_{q}^{q}
$$

where $J_{q} \in C^{1}\left(W_{0}^{s, p}(\Omega)\right)$. If

$$
\mathcal{N}_{q}(\Omega):=\left\{u \in W_{0}^{s, p}(\Omega) \backslash\{0\}:\left\langle d J_{q}(u), u\right\rangle=0\right\}
$$

is the corresponding Nehari manifold for $J_{q}$, one readily checks that

$$
c_{q}=\inf \left\{J_{q}(u): u \in \mathcal{N}_{q}(\Omega)\right\}=\left(\frac{1}{p}-\frac{1}{q}\right) \mathbb{S}_{q}(\Omega)^{\frac{q}{q-p}},
$$

and minimizers for the latter problem are (up to multiplicative constants) minimizers for (2.2). Any minimizer for (2.4) is called a ground state solution for (2.3), being a critical point of minimal energy. Following [24, Theorem 4.2] we have also the following Mountain Pass characterization of the values $c_{q}$, valid for every $q>p$

$$
c_{q}=\inf _{\substack{u \in W_{0}^{s, p}(\Omega) \\ u \neq 0}} \max _{t \geq 0} J_{q}(t u)=\inf _{\gamma \in \Gamma} \max _{t \in[0,1]} J_{q}(\gamma(t)),
$$

where

$$
\Gamma:=\left\{\gamma \in C^{0}\left([0,1], W_{0}^{s, p}(\Omega)\right): \gamma(0)=0, J_{q}(\gamma(1))<0\right\} .
$$

Any ground state solution must be of constant sign, since it also solves problem (2.2), and

$$
[|u|]_{s, p}<[u]_{s, p} \quad \text { if } u_{+}, u_{-} \neq 0 .
$$

A more precise statement is given in the following proposition (see also [6, Lemma 2.13])

Lemma 2.2 (Energy doubling). Let $q \geq p$ and $u \in W_{0}^{s, p}(\Omega)$ be a sign-changing weak solution to

$$
\begin{cases}\left(-\Delta_{p}\right)^{s} u=|u|^{q-2} u & \text { in } \Omega \\ u=0 & \text { in } \mathbb{R}^{N} \backslash \Omega\end{cases}
$$

where $\Omega$ is an arbitrary open subset of $\mathbb{R}^{N}$. Then

$$
J_{q}(u)>2\left(\frac{1}{p}-\frac{1}{q}\right) \mathbb{S}_{q}(\Omega)^{\frac{q}{q-p}} .
$$

Proof. If $u_{ \pm}:=\max \{ \pm u, 0\} \in W_{0}^{s, p}(\Omega) \backslash\{0\}$, then

$$
\pm(u(x)-u(y))^{p-1}\left(u_{ \pm}(x)-u_{ \pm}(y)\right) \geq\left|u_{ \pm}(x)-u_{ \pm}(y)\right|^{p},
$$

for a.e. $x, y \in \mathbb{R}^{N}$. Indeed, for the inequality involving the positive part, we have

$$
\begin{aligned}
& (u(x)-u(y))^{p-1}\left(u_{+}(x)-u_{+}(y)\right)=\left|u_{+}(x)-u_{+}(y)\right|^{p} \chi_{\{u(x) \geq 0, u(y) \geq 0\}} \\
& +\left(u_{+}(x)+u_{-}(y)\right)^{p-1} u_{+}(x) \chi_{\{u(x)>0, u(y)<0\}}+\left(u_{-}(x)+u_{+}(y)\right)^{p-1} u_{+}(y) \chi_{\{u(x)<0, u(y)>0\}} \\
& \geq\left|u_{+}(x)-u_{+}(y)\right|^{p} .
\end{aligned}
$$


A similar justification holds for the inequality involving the negative part. This also shows that the above inequalities are strict as long as $u_{ \pm} \neq 0$. Then, testing problem (2.5) by $\pm u_{ \pm}$ yields

$$
\left[u_{ \pm}\right]_{s, p}^{p} \leq \int_{\mathbb{R}^{2 N}} \frac{ \pm(u(x)-u(y))^{p-1}\left(u_{ \pm}(x)-u_{ \pm}(y)\right)}{|x-y|^{N+s p}} d x d y=\int_{\Omega} u_{ \pm}^{q} d x
$$

with strict inequality if $u_{ \pm} \neq 0$. Now, letting

$$
\lambda_{ \pm}:=\left(\frac{\left[u_{ \pm}\right]_{s, p}^{p}}{\left|u_{ \pm}\right|_{q}^{q}}\right)^{\frac{1}{q-p}}
$$

it holds $\lambda_{ \pm} u_{ \pm} \in \mathcal{N}_{q}(\Omega)$ and $\lambda_{ \pm}<1$. On the other hand

$$
\begin{aligned}
J_{q}(u) & =\left(\frac{1}{p}-\frac{1}{q}\right)|u|_{q}^{q}=\left(\frac{1}{p}-\frac{1}{q}\right) \int_{\Omega}\left(u_{+}^{q}+u_{-}^{q}\right) d x=\frac{1}{\lambda_{+}^{q}} J_{q}\left(\lambda_{+} u_{+}\right)+\frac{1}{\lambda_{-}^{q}} J_{q}\left(\lambda_{-} u_{-}\right) \\
& >2\left(\frac{1}{p}-\frac{1}{q}\right) \mathbb{S}_{q}(\Omega)^{\frac{q}{q-p}}
\end{aligned}
$$

which completes the proof.

We will choose in the following the nonnegative ground states, which actually turn out to be strictly positive in $\Omega$ by the following result.

Lemma 2.3 (Strong Maximum Principle). Let $u \in W_{0}^{s, p}(\Omega)$ satisfy

$$
\begin{cases}\left(-\Delta_{p}\right)^{s} u \geq 0 & \text { weakly in } \Omega, \\ u \geq 0 & \text { in } \Omega^{c} .\end{cases}
$$

Then $u$ has a lower semi-continuous representative in $\Omega$, which is either identically 0 or positive.

Proof. By the comparison principle of [14, Proposition 2.10], we get $u \geq 0$ a.e. in $\mathbb{R}^{N}$. Proceeding as in [13, Theorem 2.4] we find that $u$ admits a l.s.c. representative, which to ease the notation we will identify with $u$. Then, the set $\{x \in \Omega: u(x)=0\}$ is closed in $\Omega$. By the weak Harnack inequality [14, Theorem 5.2], it holds

$$
\inf _{B_{R / 4}} u \geq \sigma\left(f_{B_{R} \backslash B_{R / 2}} u^{p-1} d x\right)^{\frac{1}{p-1}}, \quad \forall B_{R} \subseteq \Omega .
$$

which implies that $\{x \in \Omega: u(x)=0\}$ is open in $\Omega$. Suppose $u \neq 0$, and let $\Omega=\cup_{j \in \mathcal{J}} \Omega_{j}$ where $\Omega_{j}$ are the connected components of $\Omega$. It follows from the previous discussion that for each $j \in \mathcal{J}$, either $u$ is strictly positive everywhere in $\Omega_{j}$ or it vanishes identically. Since $u \neq 0$, there is a connected component, say, $\Omega_{1}$ such that $u>0$ in $\Omega_{1}$. Suppose now by contradiction that there exists another connected component, say, $\Omega_{2}$, such that $u \equiv 0$ in $\Omega_{2}$, and let $\varphi \in C_{c}^{\infty}\left(\Omega_{2}\right), \varphi \geq 0, \varphi \neq 0$. Testing (2.6) with $\varphi$ we get

$$
0 \leq \int_{\mathbb{R}^{2 N}} \frac{(u(x)-u(y))^{p-1}(\varphi(x)-\varphi(y))}{|x-y|^{N+p s}} d x d y=-2 \int_{\Omega_{2}} \varphi(x) \int_{\Omega_{2}^{c}} \frac{u^{p-1}(y)}{|x-y|^{N+p s}} d y d x<0,
$$

since $u>0$ in $\Omega_{1} \subseteq \Omega_{2}^{c}$.

Remark 2.4. A similar statement is provided in [3, Theorem A.1] with a different proof. Notice that, contrary to the local case $s=1$, connectedness of $\Omega$ is not required. This is a typical feature of nonlocal problems, which was first outlined in [5]. 
2.3. Concentration-Compactness. We now prove a concentration-compactness lemma which was first stated without proof by P.L. Lions in [17, Remark I.6]. We say that a sequence of functions $\left\{f_{n}\right\}_{n} \subseteq L^{1}\left(\mathbb{R}^{N}\right)$ converges tightly to a Borel regular measure $d \mu$ if

$$
\forall \varphi \in C_{b}\left(\mathbb{R}^{N}\right), \quad \int_{\mathbb{R}^{N}} \varphi f_{n} d x \rightarrow \int_{\mathbb{R}^{N}} \varphi d \mu,
$$

where $C_{b}\left(\mathbb{R}^{N}\right)$ is the Banach space of bounded continuous functions on $\mathbb{R}^{N}$. Notice that this convergence is stronger than the usual weak convergence of measures as linear functionals on the separable space $C_{0}\left(\mathbb{R}^{N}\right)$ : indeed boundedness of $\left\{\left|f_{n}\right|_{1}\right\}$ does not suffice to the sequential compactness with respect to tight convergence. Nevertheless, we will still denote by with the symbol $\stackrel{*}{\rightarrow}$ the notion of tight convergence. Prokhorov theorem ensures that bounded sequences $\left\{f_{n}\right\}_{n}$ are relatively sequentially compact if and only if the sequence is tight in the sense that

$$
\forall \varepsilon>0 \exists A \subseteq \mathbb{R}^{N}: \sup _{n} \int_{A^{c}}\left|f_{n}\right| d x<\varepsilon .
$$

Theorem 2.5. Let $\left\{u_{n}\right\}$ be a bounded sequence in $W_{0}^{s, p}(\Omega)$. Then, up to a subsequence, there exists $u \in W_{0}^{s, p}(\Omega)$, two Borel regular measures $\mu$ and $\nu, \Lambda$ denumerable, $x_{j} \in \bar{\Omega}, \nu_{j} \geq 0$, $\mu_{j} \geq 0$ with $\nu_{j}+\mu_{j}>0 j \in \Lambda$, such that

$$
\begin{aligned}
& u_{n} \rightarrow u \quad \text { weakly in } W_{0}^{s, p}(\Omega) \text { and strongly in } L^{p}(\Omega), \\
& \left|D^{s} u_{n}\right|^{p} \stackrel{*}{\rightarrow} d \mu, \quad\left|u_{n}\right|^{p^{*}} \stackrel{*}{\rightarrow} d \nu \\
& d \mu \geq\left|D^{s} u\right|^{p}+\sum_{j \in \Lambda} \mu_{j} \delta_{x_{j}}, \quad \mu_{j}:=\mu\left(\left\{x_{j}\right\}\right), \\
& d \nu=|u|^{p^{*}}+\sum_{j \in \Lambda} \nu_{j} \delta_{x_{j}}, \quad \nu_{j}:=\nu\left(\left\{x_{j}\right\}\right), \\
& \mu_{j} \geq \mathbb{S} \nu_{j}^{\frac{p}{p^{*}}} .
\end{aligned}
$$

We will need the following lemma.

Lemma 2.6. Let $N>$ ps. For any $u \in L^{p^{*}}\left(\mathbb{R}^{N}\right)$ it holds

$$
\lim _{\delta \downarrow 0} \delta^{N} \int_{B_{\delta}^{c}} \frac{|u|^{p}}{|x|^{N+p s}} d x=0 .
$$

Proof. If $u \in L^{\infty}\left(\mathbb{R}^{N}\right)$, the assertion immediately follows, since a direct computation yields

$$
\delta^{N} \int_{B_{\delta}^{c}} \frac{|u|^{p}}{|x|^{N+p s}} d x \leq C\|u\|_{L^{\infty}}^{p} \delta^{N-s p} .
$$

In the general case, let $\left\{u_{k}\right\} \subset C_{c}^{\infty}\left(\mathbb{R}^{N}\right)$ be such that $u_{k} \rightarrow u$ in $L^{p^{*}}\left(\mathbb{R}^{N}\right)$ as $k \rightarrow \infty$. By using Hölder inequality, for any $\delta>0$ and $k \in \mathbb{N}$, we obtain

$$
\begin{aligned}
\delta^{N} \int_{B_{\delta}^{c}} \frac{|u|^{p}}{|x|^{N+p s}} d x & \leq C \delta^{N} \int_{B_{\delta}^{c}} \frac{\left|u_{k}-u\right|^{p}}{|x|^{N+p s}} d x+C \delta^{N} \int_{B_{\delta}^{c}} \frac{\left|u_{k}\right|^{p}}{|x|^{N+p s}} d x \\
& \leq C\left|u_{k}-u\right|_{p^{*}}^{p}+C \delta^{N} \int_{B_{\delta}^{c}} \frac{\left|u_{k}\right|^{p}}{|x|^{N+p s}} d x .
\end{aligned}
$$

Since any $u_{k}$ is bounded, letting $\delta \rightarrow 0$ yields

$$
\limsup _{\delta \rightarrow 0} \delta^{N} \int_{B_{\delta}^{c}} \frac{|u|^{p}}{|x|^{N+p s}} d x \leq C\left|u_{k}-u\right|_{p^{*}}^{p} .
$$

Finally, letting $k \rightarrow \infty$ concludes the proof. 
Now we proceed proving Theorem 2.5

Proof. Since $\Omega$ is bounded and $u_{n} \equiv 0$ in $\Omega^{c}$, the sequence $\left\{\left|u_{n}\right|^{p^{*}}\right\}$ is tight, ensuring the existence of $\nu$ (and clearly $\operatorname{supp}(\nu) \subseteq \bar{\Omega}$ ). To prove the tightness of $\left\{\left|D^{s} u_{n}\right|^{p}\right\}$, let $U$ be open and bounded such that $U \supset \bar{\Omega}$. If $\operatorname{dist}\left(U^{c}, \Omega\right)=: \theta>0$, then for any $x \in U^{c}$ and $y \in \Omega$ it holds $|x-y| \geq C_{\theta}|x|$, and thus

$$
\left|D^{s} u_{n}\right|^{p}(x)=\int_{\Omega} \frac{\left|u_{n}(y)\right|^{p}}{|x-y|^{N+p s}} d y \leq C \frac{\left[u_{n}\right]_{s, p}^{p}}{|x|^{N+p s}}, \quad \text { for a.e. } x \in U^{c} .
$$

The latter inequality readily implies tightness of $\left\{\left|D^{s} u_{n}\right|\right\}$ and thus (2.7) is proved. We come to the proof of (2.8), (2.9) and (2.10). We shall follow the proof of [17, Lemma I.1], by supposing first that $u \equiv 0$. From Sobolev's inequality and (2.1), we have, for any $\varphi \in C_{c}^{\infty}\left(\mathbb{R}^{N}\right)$,

$$
\mathbb{S}\left|u_{n} \varphi\right|_{p^{*}}^{\frac{p}{p^{*}}} \leq\left[u_{n} \varphi\right]_{s, p}^{p} \leq(1+\theta) \int_{\mathbb{R}^{N}}\left|D^{s} u_{n}\right|^{p}|\varphi|^{p} d x+C_{\theta} \int_{\mathbb{R}^{N}}\left|D^{s} \varphi\right|^{p}\left|u_{n}\right|^{p} d x .
$$

Letting $n \rightarrow+\infty$ and using that $u_{n} \rightarrow 0$ in $L^{p}\left(\mathbb{R}^{N}\right)$ and $\left|D^{s} \varphi\right|^{p} \in L^{\infty}\left(\mathbb{R}^{N}\right)$, we obtain

$$
\mathbb{S}\left(\int_{\mathbb{R}^{N}}|\varphi|^{p^{*}} d \nu\right)^{\frac{p}{p^{*}}} \leq(1+\theta) \int_{\mathbb{R}^{N}}|\varphi|^{p} d \mu
$$

Letting $\theta \downarrow 0$ proves (2.8) and (2.9), due to [17, Lemma I.2]. Finally, the previous inequality easily implies, for any $j \in \Lambda$

$$
\mathbb{S}\left(\nu\left(B_{\delta}\left(x_{j}\right)\right)\right)^{\frac{p}{p^{*}}} \leq(1+\theta) \mu\left(B_{2 \delta}\left(x_{j}\right)\right),
$$

which provides (2.10) taking the limit for $\theta \downarrow 0$ and then $\delta \downarrow 0$. To prove the case $u \neq 0$, one can proceed as in [17] to obtain (2.9). Concerning (2.8), we first claim that $d \mu \geq\left|D^{s} u\right|^{p}$. Indeed, for any $\varphi \in C_{c}^{\infty}\left(\mathbb{R}^{N}\right), \varphi \geq 0$, the functional

$$
v \mapsto \int_{\mathbb{R}^{N}}\left|D^{s} v\right|^{p} \varphi d x
$$

is convex and continuous, therefore $u_{n} \rightarrow u$ in $W_{0}^{s, p}(\Omega)$ implies

$$
\int_{\mathbb{R}^{N}} \varphi d \mu=\lim _{n \rightarrow \infty} \int_{\mathbb{R}^{N}}\left|D^{s} u_{n}\right|^{p} \varphi d x \geq \int_{\mathbb{R}^{N}}\left|D^{s} u\right|^{p} \varphi d x, \quad \text { for any } \varphi \in C_{c}^{\infty}\left(\mathbb{R}^{N}\right), \quad \varphi \geq 0 .
$$

On the other hand (2.12) implies $\mu\left(\left\{x_{j}\right\}\right)>0$ whenever $\nu\left(\left\{x_{j}\right\}\right)>0$. Then (2.8) follows. We come to (2.10). We take the limit for $n \rightarrow+\infty$ in (2.11) to obtain

$$
\mathbb{S}\left(\int_{\mathbb{R}^{N}}|\varphi|^{p^{*}} d \nu\right)^{\frac{p}{p^{*}}} \leq(1+\theta) \int_{\mathbb{R}^{N}}|\varphi|^{p} d \mu+C_{\theta} \int_{\mathbb{R}^{N}}\left|D^{s} \varphi\right|^{p}|u|^{p} d x
$$

Now we fix $x_{j}$, and for any $\delta>0$, let $\varphi_{\delta} \in C_{c}^{\infty}\left(B_{2 \delta}\left(x_{j}\right)\right)$ satisfy

$$
0 \leq \varphi_{\delta}, \quad \varphi\left\lfloor_{B_{\delta}}=1, \quad\left|\varphi_{\delta}\right|_{\infty} \leq 1, \quad\left|\nabla \varphi_{\delta}\right|_{\infty} \leq C / \delta .\right.
$$

We claim the following:

$$
\forall u \in L^{p^{*}}\left(\mathbb{R}^{N}\right), \quad \lim _{\delta \downarrow 0} \int_{\mathbb{R}^{2 N}}|u|^{p}\left|D^{s} \varphi_{\delta}\right|^{p} d x=0 .
$$

Without loss of generality, suppose $x_{j}=0$. Let $A=\left(B_{2 \delta} \times B_{2 \delta}\right) \cup\left(B_{\delta} \times B_{2 \delta}^{c}\right) \cup\left(B_{2 \delta}^{c} \times B_{\delta}\right)$ and notice that on $\mathbb{R}^{2 N} \backslash A$ it holds $\left|\varphi_{\delta}(x)-\varphi_{\delta}(y)\right|=0$. Then on each of the three pieces 
forming $A$ we proceed as follows. Since $\left|\varphi_{\delta}(x)-\varphi_{\delta}(y)\right| \leq C \delta^{-1}|x-y|$, on $B_{2 \delta} \times B_{2 \delta}$ we have

$$
\begin{aligned}
\int_{B_{2 \delta} \times B_{2 \delta}} \frac{|u(y)|^{p}\left|\varphi_{\delta}(x)-\varphi_{\delta}(y)\right|^{p}}{|x-y|^{N+p s}} d x d y & \leq \int_{B_{2 \delta} \times B_{4 \delta}}|u(y)|^{p} \frac{C^{p} \delta^{-p}|z|^{p}}{|z|^{N+p s}} d y d z \\
& \leq \frac{C}{\delta^{p s}}\left(\int_{B_{2 \delta}}|u|^{p^{*}} d y\right)^{\frac{p}{p^{*}}}\left|B_{2 \delta}\right|^{1-\frac{p}{p^{*}}}=C\left(\int_{B_{2 \delta}}|u|^{p^{*}} d y\right)^{\frac{p}{p^{*}}}
\end{aligned}
$$

which vanishes as $\delta \downarrow 0$. On $B_{2 \delta}^{c} \times B_{\delta}$, the triangle inequality implies that $|x-y| \geq|x| / 2$ and thus, as $\delta \downarrow 0$,

$$
\begin{aligned}
\int_{B_{2 \delta}^{c} \times B_{\delta}} \frac{|u(y)|^{p}\left|\varphi_{\delta}(x)-\varphi_{\delta}(y)\right|^{p}}{|x-y|^{N+p s}} d x d y & \leq C \int_{B_{2 \delta}^{c} \times B_{\delta}}|u(y)|^{p} \frac{1}{|x|^{N+p s}} d x d y \\
& \leq C\left(\int_{B_{\delta}}|u|^{p^{*}} d y\right)^{\frac{p}{p^{*}}}\left|B_{\delta}\right|^{1-\frac{p}{p^{*}}} \frac{1}{\delta^{p s}}=o(1) .
\end{aligned}
$$

Finally on $B_{\delta} \times B_{2 \delta}^{c}$ it holds $|x-y| \geq|y| / 2$ and thus

$$
\int_{B_{\delta} \times B_{2 \delta}^{c}} \frac{|u(y)|^{p}\left|\varphi_{\delta}(x)-\varphi_{\delta}(y)\right|^{p}}{|x-y|^{N+p s}} d x d y \leq C \delta^{N} \int_{B_{2 \delta}^{c}} \frac{|u(y)|^{p}}{|y|^{N+p s}} d y
$$

which vanishes as $\delta \downarrow 0$ by the previous lemma, and this proves (2.14). Now if $\varphi_{\delta}=1$ in $B_{\delta}$ we obtain

$$
\mathbb{S}\left(\nu\left(B_{\delta}\right)\right)^{\frac{p}{p^{*}}} \leq(1+\theta) \mu\left(B_{2 \delta}\right)+C_{\theta} o(1)
$$

which gives (2.10) taking $\theta \downarrow 0$ and then $\delta \downarrow 0$.

\section{Limiting BeHAViour FOR $\varepsilon \rightarrow 0$}

The next result provides lower bounds for the masses $\mu_{j}$ and $\nu_{j}$ given by the ConcentrationCompactness theorem.

Lemma 3.1. Let $u_{\varepsilon}$ solve (1.1), and $\nu_{j}, \mu_{j}$ be as in (2.8)-(2.9). Then, for any $j \in \Lambda$,

$$
\mu_{j} \geq \mathbb{S}^{\frac{N}{s p}}, \quad \nu_{j} \geq \mathbb{S}^{\frac{N}{s p}} .
$$

Proof. Suppose again that $x_{j}=0$ and choose $\varphi_{\delta} \in C_{c}^{\infty}\left(B_{2 \delta}\right)$ as in formula (2.13). Testing the equation with $\varphi_{\delta} u_{\varepsilon}$, we get

$$
\begin{aligned}
0= & \int_{\mathbb{R}^{2 N}} \frac{\left(u_{\varepsilon}(x)-u_{\varepsilon}(y)\right)^{p-1}\left(\varphi_{\delta}(x) u_{\varepsilon}(x)-\varphi_{\delta}(y) u_{\varepsilon}(y)\right)}{|x-y|^{N+p s}} d x d y-\int_{\mathbb{R}^{N}} u_{\varepsilon}^{p^{*}-\varepsilon} \varphi_{\delta} d x \\
\geq & \int_{\mathbb{R}^{N}}\left|D^{s} u_{\varepsilon}\right|^{p} \varphi_{\delta} d x-\left(\int_{\mathbb{R}^{N}}\left|u_{\varepsilon}\right|^{p^{*}} \varphi_{\delta} d x\right)^{1-\frac{\varepsilon}{p^{*}}}\left|B_{2 \delta}\left(x_{j}\right)\right|^{\frac{\varepsilon}{p^{*}}} \\
& +\int_{\mathbb{R}^{2 N}} \frac{\left(u_{\varepsilon}(x)-u_{\varepsilon}(y)\right)^{p-1} u_{\varepsilon}(y)\left(\varphi_{\delta}(x)-\varphi_{\delta}(y)\right)}{|x-y|^{N+p s}} d x d y .
\end{aligned}
$$

Moreover, by Hölder's inequality, we get

$$
\left|\int_{\mathbb{R}^{2 N}} \frac{\left(u_{\varepsilon}(x)-u_{\varepsilon}(y)\right)^{p-1} u_{\varepsilon}(y)\left(\varphi_{\delta}(x)-\varphi_{\delta}(y)\right)}{|x-y|^{N+p s}} d x d y\right| \leq\left[u_{\varepsilon}\right]_{s, p}^{p-1}\left(\int_{\mathbb{R}^{N}}\left|D^{s} \varphi_{\delta}\right|^{p}\left|u_{\varepsilon}\right|^{p} d y\right)^{\frac{1}{p}} .
$$

Notice that $\left|D^{s} \varphi_{\delta}\right|^{p} \in L^{\infty}\left(\mathbb{R}^{N}\right)$, since

$$
\int_{\mathbb{R}^{N}} \frac{\left|\varphi_{\delta}(x)-\varphi_{\delta}(y)\right|^{p}}{|x-y|^{N+p s}} d y \leq \frac{C}{\delta^{p}} \int_{\mathbb{R}^{N}} \frac{\min \left\{1,|x-y|^{p}\right\}}{|x-y|^{N+p s}} d y \leq \frac{C}{\delta^{p}} .
$$


Up to subsequences we can suppose that $u_{\varepsilon} \rightarrow u \in L^{p}(\Omega)$ as $\varepsilon \rightarrow 0$ and, thus,

$$
\varlimsup_{\varepsilon \rightarrow 0}\left|\int_{\mathbb{R}^{2 N}} \frac{\left(u_{\varepsilon}(x)-u_{\varepsilon}(y)\right)^{p-1} u_{\varepsilon}(y)\left(\varphi_{\delta}(x)-\varphi_{\delta}(y)\right)}{|x-y|^{N+p s}} d x d y\right| \leq C\left(\int_{\mathbb{R}^{N}}\left|D^{s} \varphi_{\delta}\right|^{p}|u|^{p} d y\right)^{\frac{1}{p}} .
$$

Taking the limit for $\varepsilon \rightarrow 0$ in (3.2) we therefore obtained

$$
\int_{\mathbb{R}^{N}} \varphi_{\delta} d \mu \leq \int_{\mathbb{R}^{N}} \varphi_{\delta} d \nu+C\left(\int_{\mathbb{R}^{N}}\left|D^{s} \varphi_{\delta}\right|^{p}|u|^{p} d y\right)^{\frac{1}{p}}
$$

or, by (2.14),

$$
\int_{\mathbb{R}^{N}} \varphi_{\delta} d \mu \leq \int_{\mathbb{R}^{N}} \varphi_{\delta} d \nu+o(1)
$$

with $o(1) \rightarrow 0$ as $\delta \downarrow 0$. This implies $\nu_{j} \geq \mu_{j}$, which, coupled together with (2.10), gives (3.1).

Remark 3.2. From Lemma 3.1, the concentration points for $\nu$ agree with those for $\mu$ and, being $\nu$ of finite mass, the concentration set is finite, say $\mathscr{C}:=\left\{x_{1}, \ldots, x_{M}\right\}$, for some $M \in \mathbb{N}$.

Let now $V$ be an optimizer for the Sobolev constant $\mathbb{S}$ which solves (1.1) with $\varepsilon=0$. For $\delta>0$, we define the functions

$$
V_{\delta}(x):=\frac{1}{\delta^{(N-s p) / p}} V\left(\frac{|x|}{\delta}\right)
$$

and consider

$$
m_{\delta}:=\frac{V_{\delta}(1)}{V_{\delta}(1)-V_{\delta}(\theta)}, \quad G_{\delta}(t):= \begin{cases}0, & 0 \leq t \leq V_{\delta}(\theta) \\ m_{\delta}\left(t-V_{\delta}(\theta)\right), & V_{\delta}(\theta) \leq t \leq V_{\delta}(1) \\ t, & t \geq V_{\delta}(1)\end{cases}
$$

where $\theta>1$ is a suitable constant introduced in [19] and the radially symmetric function

$$
v_{\delta}(r)=G_{\delta}\left(V_{\delta}(r)\right)= \begin{cases}V_{\delta}(r), & r \leq 1 \\ 0, & r \geq \theta\end{cases}
$$

We have the following estimates.

Lemma 3.3. There exists a constant $C=C(N, p, s)>0$ such that

$$
\begin{aligned}
{\left[v_{\delta}\right]_{s, p}^{p} } & \leq \mathbb{S}^{\frac{N}{p s}}+C \delta^{\frac{N-p s}{p-1}}, \\
\int_{\mathbb{R}^{N}} v_{\delta}^{p^{*}-\varepsilon} d x & \geq \int_{\mathbb{R}^{N}} V_{\delta}(x)^{p^{*}-\varepsilon} d x-C \delta^{\left(p^{*}-\varepsilon\right) \frac{N-p s}{p(p-1)}}
\end{aligned}
$$

for any $\delta \leq 1 / 2$ and $\varepsilon<N /(N-s p)$.

Proof. The first inequality was proved in [19]. Concerning the second inequality, we have

$$
\int_{\mathbb{R}^{N}} v_{\delta}^{p^{*}-\varepsilon} d x \geq \int_{B_{1}} V_{\delta}(x)^{p^{*}-\varepsilon} d x=\int_{\mathbb{R}^{N}} V_{\delta}(x)^{p^{*}-\varepsilon} d x-\int_{B_{1}^{c}} V_{\delta}(x)^{p^{*}-\varepsilon} d x
$$

By virtue of $[4$, Theorem 1.1], we have

$$
\begin{aligned}
\int_{B_{1}^{c}} V_{\delta}(x)^{p^{*}-\varepsilon} d x & =\delta^{\frac{N-s p}{p} \varepsilon} \int_{B_{1 / \delta}^{c}} V(x)^{p^{*}-\varepsilon} d x \\
& \leq C \delta^{\frac{N-s p}{p} \varepsilon} \int_{B_{1 / \delta}^{c}} \frac{1}{|y|^{\frac{N-s p}{p-1}\left(p^{*}-\varepsilon\right)}} d y \leq C \delta^{\frac{(N-s p)\left(p^{*}-\varepsilon\right)}{p(p-1)}},
\end{aligned}
$$

which concludes the proof. 
Theorem 3.4. Let $\left\{u_{n}\right\}_{n}$ be a sequence of ground state solutions to (1.1) for some $\varepsilon_{n} \downarrow 0$. Then

$$
\lim _{n} I_{\varepsilon_{n}}\left(u_{n}\right)=\frac{s}{N} \mathbb{S}^{N / p s}
$$

Proof. By testing the equation by $u_{n}$ and using Hölder and Sobolev inequality we get

$$
\left[u_{n}\right]_{s, p}^{p} \geq \mathbb{S} \frac{p^{*}-\varepsilon_{n}}{p^{*}-p-\varepsilon_{n}}|\Omega|^{-\frac{\varepsilon_{n} p}{\left(p^{*}-p-\varepsilon_{n}\right) p^{*}}}
$$

Therefore, we have

$$
I_{\varepsilon_{n}}\left(u_{n}\right)=\left(\frac{1}{p}-\frac{1}{p^{*}-\varepsilon_{n}}\right)\left[u_{n}\right]_{s, p}^{p} \geq\left(\frac{1}{p}-\frac{1}{p^{*}-\varepsilon_{n}}\right) \mathbb{S}^{\frac{p^{*}-\varepsilon_{n}}{p^{*}-p-\varepsilon_{n}}}|\Omega|^{-\frac{\varepsilon_{n} p}{\left(p^{*}-p-\varepsilon_{n}\right) p^{*}}}
$$

yielding

$$
\lim _{n} I_{\varepsilon_{n}}\left(u_{n}\right) \geq \frac{s}{N} \mathbb{S}^{\frac{N}{p s}}
$$

To prove the opposite inequality, suppose without loss of generality that $\bar{B}_{\theta} \subseteq \Omega$. Since $\lim _{t \rightarrow+\infty} I_{\varepsilon_{n}}\left(t v_{\delta}\right)=-\infty$, by the definition of the mountain pass level it holds

$$
I_{\varepsilon_{n}}\left(u_{n}\right) \leq \sup _{t>0} I_{\varepsilon_{n}}\left(t v_{\delta}\right)=\left(\frac{1}{p}-\frac{1}{p^{*}-\varepsilon_{n}}\right)\left(\frac{\left[v_{\delta}\right]_{s, p}}{\left|v_{\delta}\right|_{p^{*}-\varepsilon_{n}}}\right)^{\frac{p^{*}-\varepsilon_{n}}{p^{*}-p-\varepsilon_{n}}} .
$$

Using the previous lemma we thus obtain

$$
\varlimsup_{n} I_{\varepsilon_{n}}\left(u_{n}\right) \leq \frac{s}{N} \varlimsup_{n} \frac{\left(\mathbb{S}^{\frac{N}{p s}}+C \delta^{\frac{N-p s}{p-1}}\right)^{\frac{p^{*}-\varepsilon_{n}}{p^{*}-p-\varepsilon_{n}}}}{\left(\left|V_{\delta}\right|_{p^{*}-\varepsilon_{n}}^{p^{*}-\varepsilon_{n}}-C \delta^{\left.\left(p^{*}-\varepsilon_{n}\right) \frac{N-p s}{p(p-1)}\right)^{\frac{p}{p^{*}-p-\varepsilon_{n}}}}\right.} .
$$

Now by dominated convergence, we get

$$
\lim _{n}\left|V_{\delta}\right|_{p^{*}-\varepsilon_{n}}^{p^{*}-\varepsilon_{n}}=\left|V_{\delta}\right|_{p^{*}}^{p^{*}}=\mathbb{S}^{\frac{N}{p s}}
$$

and therefore

$$
\varlimsup_{n} I_{\varepsilon_{n}}\left(u_{n}\right) \leq \frac{s}{N} \frac{\left(\mathbb{S}^{\frac{N}{p s}}+C \delta^{\frac{N-p s}{p-1}}\right)^{\frac{p^{*}}{p^{*}-p}}}{\left(\mathbb{S}^{\frac{N}{p s}}-C \delta^{\frac{N}{p-1}}\right)^{\frac{p}{p^{*}-p}}} \leq \frac{s}{N} \mathbb{S}^{\frac{N}{p s}}\left(1+C \delta^{\frac{N-p s}{p-1}}\right)
$$

for some universal constant $C=C(N, p, s)$. Letting $\delta \rightarrow 0$ proves the claim.

Proof of Theorem 1.1. The proof follows from (3.1) and Theorem 3.4 exactly as in [11], providing us with $\bar{x} \in \bar{\Omega}$ satisfying both conditions.

As a direct application we have the following multiplicity result on annular domains.

Theorem 3.5. Let $\Omega=B_{R} \backslash B_{r}$ for some $R>r>0$. Then for $q<p^{*}$ sufficiently near $p^{*}$ there is a continuum of positive solutions to

$$
\begin{cases}\left(-\Delta_{p}\right)^{s} u=u^{q} & \text { in } \Omega, \\ u=0 & \text { in } \Omega^{c} .\end{cases}
$$

Proof. Suppose not. Then there is a sequence $\varepsilon_{n} \downarrow 0$ such that there is only a denumerable set of positive solutions to (1.1) for any $n$. Let $q=p^{*}-\varepsilon_{n}$ and $\left\{u_{n}\right\}_{n}$ be a nonnegative ground state for (1.1), which by the Strong Maximum Principle is strictly positive in $\Omega$. 
Then, by virtue of Theorem 1.1, there exists a point $\bar{x} \in \bar{\Omega}$ such that, up to a subsequence, $\left|u_{n}\right|^{p^{*}} \stackrel{*}{\rightarrow} \mathbb{S}^{\frac{N}{p s}} \delta_{\bar{x}}$. For sufficently large $n$ it thus holds

$$
\int_{\mathbb{R}^{N}}\left|u_{n}\right|^{p^{*}} d x<\frac{3}{2} \mathbb{S}^{\frac{N}{p s}}, \quad \int_{B_{r}(\bar{x})}\left|u_{n}\right|^{p^{*}} d x>\frac{3}{4} \mathbb{S}^{\frac{N}{p s}} .
$$

For any such $\varepsilon_{n} \rightarrow 0$, the solution $u_{n}$ eventually cannot be radial since otherwise also the integral on $B_{r}(-\bar{x})$ (disjoint from $B_{r}(\bar{x})$ as $|\bar{x}| \geq r$ ) would be greater than $3 \mathbb{S}^{N / p s} / 4$, thus yielding

$$
\frac{3}{2} \mathbb{S}^{\frac{N}{p s}}>\int_{\mathbb{R}^{N}}\left|u_{n}\right|^{p^{*}} d x \geq \int_{B_{r}(\bar{x})}\left|u_{n}\right|^{p^{*}} d x+\int_{B_{r}(-\bar{x})}\left|u_{n}\right|^{p^{*}} d x>\frac{3}{2} \mathbb{S}^{\frac{N}{p s}}
$$

for sufficiently large $n=n_{0} \in \mathbb{N}$. The map

$$
R \in \mathrm{SO}_{N} \rightarrow W_{0}^{s, p}(\Omega) \ni u_{n_{0}} \circ R
$$

is therefore a continuous, non-constant map, all of whose image is made of positive solutions to (1.1). This gives the contradiction.

Lemma 3.6. Let $\Omega$ be a bounded domain and $\left\{u_{n}\right\}_{n}$ be a sequence of ground state solutions to (1.1) for $\varepsilon_{n} \downarrow 0$ such that $I_{\varepsilon_{n}}\left(u_{n}\right)$ is bounded and Theorem 2.5 holds, with u being its weak limit. Then if $u \neq 0$,

$$
\int_{\mathbb{R}^{N}}\left|D^{s} u\right|^{p} d x \geq \mathbb{S}^{\frac{N}{p s}}, \quad \int_{\mathbb{R}^{N}}|u|^{p^{*}} d x \geq \mathbb{S}^{\frac{N}{p s}}
$$

Proof. Let $\mathscr{C}=\left\{x_{1}, \ldots, x_{M}\right\}$ be the concentration set, and let $\varphi_{\delta}$ be the cut-off functions as introduced in (2.13). Define, for any $\delta>0$ small enough, the function

$$
\psi_{\delta}(x):=\sum_{i=1}^{M} \varphi_{\delta}\left(x-x_{i}\right) .
$$

Proceeding as in Lemma 3.1, testing (1.1) with $\left(1-\psi_{\delta}\right) u_{n}$ and letting $n \rightarrow \infty$, we obtain

$$
0 \geq \int_{\mathbb{R}^{N}}\left(1-\psi_{\delta}\right) d \mu-\int_{\mathbb{R}^{N}}\left(1-\psi_{\delta}\right) d \nu-o(1), \quad \text { with } o(1) \rightarrow 0 \text { as } \delta \downarrow 0 .
$$

Since $\psi_{\delta}=1$ on $\bigcup_{i=1}^{M} B_{\delta}\left(x_{i}\right)$ and $\psi_{\delta}=0$ on $\bigcap_{i=1}^{M} B_{2 \delta}^{c}\left(x_{i}\right)$, we get

$$
\lim _{\delta \downarrow 0} \int_{\mathbb{R}^{N}}\left(1-\psi_{\delta}\right) d \nu=\nu\left(\mathbb{R}^{N} \backslash \mathscr{C}\right), \quad \lim _{\delta \downarrow 0} \int_{\mathbb{R}^{N}}\left(1-\psi_{\delta}\right) d \mu=\mu\left(\mathbb{R}^{N} \backslash \mathscr{C}\right),
$$

yielding in turn, by formulas (3.5) and (2.8),

$$
\int_{\mathbb{R}^{N}}|u|^{p^{*}} d x=\nu\left(\mathbb{R}^{N} \backslash \mathscr{C}\right) \geq \mu\left(\mathbb{R}^{N} \backslash \mathscr{C}\right) \geq \int_{\mathbb{R}^{N}}\left|D^{s} u\right|^{p} d x
$$

Using also Sobolev's inequality we deduce (3.4) as long as $u \neq 0$.

Proof of Theorem 1.2. By Lemma 2.2 we can suppose that $u_{n} \geq 0$, for sufficiently small $\varepsilon_{n}>0$. Let $\mu, \nu$ be given in Theorem 2.5 and let $\mathscr{C}=\left\{x_{1}, \ldots, x_{M}\right\}$ be their concentration set. If $u \neq 0$, by the previous lemma and (3.1), we have

$$
c=\lim _{\varepsilon \rightarrow 0}\left(\frac{1}{p}-\frac{1}{p^{*}-\varepsilon}\right)\left[u_{n}\right]_{s, p}^{p}=\frac{s}{N} \mu\left(\mathbb{R}^{N}\right) \geq \frac{s}{N} \int_{\mathbb{R}^{N}}\left|D^{s} u\right|^{p} d x+\frac{s}{N} \sum_{i=1}^{M} \mu_{j} \geq \frac{s}{N}(M+1) \mathbb{S}^{\frac{N}{p s}} .
$$


Therefore $M=0$ by assumption (1.3). To prove that $u$ weakly solves

$$
\begin{cases}\left(-\Delta_{p}\right)^{s} u=u^{p^{*}-1} & \text { in } \Omega, \\ u=0 & \text { in } \Omega^{c},\end{cases}
$$

it suffices to show that, up to subsequences, $f_{n}:=u_{n}^{p^{*}-1-\varepsilon_{n}}$ weakly converges to $f:=u^{p^{*}-1}$ in $L^{p^{* \prime}}\left(\mathbb{R}^{N}\right)$. By compactness in $L^{p}(\Omega)$ we can assume that $u_{n} \rightarrow u$ pointwise a.e. in $\mathbb{R}^{N}$ so that $f_{n} \rightarrow f$ pointwise a.e. in $\mathbb{R}^{N}$. Moreover

$$
\begin{aligned}
\int_{\mathbb{R}^{N}}\left|f_{n}\right|^{\frac{p^{*}}{p^{*}-1}} d x & =\int_{\Omega} u_{n}^{p^{*}-\varepsilon_{n}} \frac{p^{*}}{p^{*}-1} d x \\
& \leq\left(\int_{\Omega}\left|u_{n}\right|^{p^{*}} d x\right)^{1-\frac{\varepsilon_{n}}{p^{*}-1}}|\Omega|^{\frac{\varepsilon_{n}}{p^{*}-1}},
\end{aligned}
$$

so that $f_{n} \rightarrow f$ in $L^{p^{* \prime}}\left(\mathbb{R}^{N}\right)$ as $n \rightarrow \infty$. It follows that $u \in W_{0}^{s, p}(\Omega)$ weakly solves (3.7) in $\Omega$. We finally prove that $u_{n} \rightarrow u$ strongly in $W_{0}^{s, p}(\Omega)$. By Hölder's inequality it holds

$$
\int_{\mathbb{R}^{N}} u_{n}^{p^{*}} d x \geq\left(\int_{\mathbb{R}^{N}} u_{n}^{p^{*}-\varepsilon_{n}} d x\right)^{\frac{p^{*}}{p^{*}-\varepsilon_{n}}}|\Omega|^{-\frac{\varepsilon_{n}}{p^{*}-\varepsilon_{n}}},
$$

and thus, up to subsequences

$$
[u]_{s, p}^{p}=|u|_{p^{*}}^{p^{*}}=\nu\left(\mathbb{R}^{N}\right)=\varlimsup_{n}\left|u_{n}\right|_{p^{*}}^{p^{*}} \geq \varlimsup_{n}\left|u_{n}\right|_{p^{*}-\varepsilon_{n}}^{p^{*}}|\Omega|^{-\frac{\varepsilon_{n}}{p^{*}-\varepsilon_{n}}}=\varlimsup_{n}\left[u_{n}\right]_{s, p}^{p \frac{p^{*}}{p^{*}-\varepsilon_{n}}}=\varlimsup_{n}\left[u_{n}\right]_{s, p}^{p} .
$$

Being $W_{0}^{s, p}(\Omega)$ uniformly convex, the claim follows. Suppose on the other hand that $u=0$. From formula (2.9) we get $d \nu=\sum_{j=1}^{M} \nu_{j} \delta_{x_{j}}$ and we know that $d \mu \geq \sum_{j=1}^{M} \mu_{j} \delta_{x_{j}}$. On the other hand formula (3.3), which holds for arbitrary $\varphi \in C_{c}^{\infty}\left(\mathbb{R}^{N}\right)$ with $\varphi \geq 0$, implies $d \nu \geq d \mu$. Therefore $\operatorname{supp}(\mu) \subseteq \operatorname{supp}(\nu)=\mathscr{C}$, which yields $d \mu=\sum_{j=1}^{M} \mu_{j} \delta_{x_{j}}$. Thus (3.6) forces $M=1$ and $c=\frac{s}{N} \mu_{1}$, where $\mu_{1}>0$.

\section{THE CASE $p=2$}

Given $\Omega \subseteq \mathbb{R}^{N}$, we let in this section $\delta(x)=\operatorname{dist}\left(x, \Omega^{c}\right)$. We will prove the following

Theorem 4.1. Let $\Omega$ be a bounded $C^{1,1}$ domain. There exists a constant $d=d(\Omega)>0$ such that any weak solution of

$$
\begin{cases}(-\Delta)^{s} u=f(u) & \text { in } \Omega, \\ u=0 & \text { in } \Omega^{c}\end{cases}
$$

with $f \in \operatorname{Lip}_{\text {loc }}(\mathbb{R})$ such that

$$
0 \leq f(t) \leq C\left(1+|t|^{p^{*}}\right), \quad r \mapsto \frac{f\left(r^{N-2 s} t\right)}{r^{N+2 s}} \quad \text { is non-increasing for any } t \geq 0
$$

satisfies for any $x_{0} \in \partial \Omega$, with exterior normal $\nu$

$$
\xi_{1}<\xi_{2} \leq 2 d \Rightarrow u\left(x_{0}-\xi_{1} \nu\right)<|1+2 d|^{N-2 s} u\left(x_{0}-\xi_{2} \nu\right) .
$$

Remark 4.2. Notice that, if $u \geq 0$ a.e. in $\Omega$, then by the strong maximum principle, $u>0$ in $\Omega$ or $u \equiv 0$. Regarding the hypothesis (4.2), consider the homogeneous case $f(t)=t^{q-1}$, $N>2 s$. Then the monotonicity property required in (4.2) is satisfied if and only if $q \leq 2^{*}$. Finally, it is worth noting that the proof actually only requires that $\partial \Omega$ satisfy a uniform exterior sphere condition, so that (4.3) actually holds, e.g., in convex domains. 
The main point of the theorem is that the constant $d$ is geometric and independent on the nonlinearity, as long as (4.2) holds. Let us now show how (4.3) implies that the ground states $u_{\varepsilon}$ cannot concentrate on $\partial \Omega$. To the best of our knowledge this argument is new.

Proof of Theorem 1.3. Let $d>0$ be given in the previous theorem, so small that

$$
\Pi(x):=\operatorname{Argmin}\{|x-y|: y \in \partial \Omega\}, \quad x \in N_{t},
$$

is well defined and $C^{1,1}$, where $N_{t}:=\{x \in \bar{\Omega}: \delta(x) \leq t\}$ for any $0 \leq t \leq 2 d$. Then, denoting by $\nu_{z}$ the exterior normal to $\partial \Omega$ at $z$, the map

$$
(z, \xi) \in \partial \Omega \times[0,2 d] \rightarrow \Phi(z, \xi)=z-\xi \nu_{z} \in N_{2 d},
$$

is a bi-Lipschitz homeomorphism, with

$$
\|\operatorname{det} D \Phi\|_{\infty}+\left\|\operatorname{det} D \Phi^{-1}\right\|_{\infty} \leq L=L(\Omega) .
$$

Suppose by contradiction that $\bar{x} \in \partial \Omega$, where $\bar{x}$ is the concentration point given by Theorem 1.1. Then for any small $\theta>0$ to be defined later, there exists a sufficiently large $n \in \mathbb{N}$ such that

$$
\int_{N_{d}}\left|u_{n}\right|^{2^{*}} d x \geq \mathbb{S}^{\frac{N}{2 s}} / 2, \quad \int_{N_{2 d} \backslash N_{d}}\left|u_{n}\right|^{2^{*}} d x<\theta .
$$

Using the change of variables given by $\Phi$, the bound (4.4) and (4.3), we have

$$
\begin{aligned}
\int_{N_{d}}\left|u_{n}\right|^{2^{*}} d x & \leq L \int_{\partial \Omega \times[0, d]}\left|u_{n}\right|^{2^{*}}\left(z-\xi \nu_{z}\right) d z d \xi \\
& \leq|1+2 d|^{2 N} L \int_{\partial \Omega \times[d, 2 d]}\left|u_{n}\right|^{2^{*}}\left(z-\xi \nu_{z}\right) d z d \xi \\
& \leq|1+2 d|^{2 N} L^{2} \int_{N_{2 d} \backslash N_{d}}\left|u_{n}\right|^{2^{*}} d x .
\end{aligned}
$$

So that, choosing $\theta|1+2 d|^{2 N} L^{2}<\mathbb{S}^{\frac{N}{2 s}} / 2$, we get a contradiction.

Proof of Theorem 4.1. The proof relies on the moving plane method, performed after suitable Kelvin transforms through externally tangent balls. First of all, being $\Omega$ a $C^{1,1}$ bounded domain, there exists $r>0$ such that at any point $x_{0} \in \partial \Omega$ there exists an open ball $B_{r}$ of radius $r$ with

$$
B_{r} \subseteq \Omega^{c}, \quad \partial B_{r} \cap \partial \Omega=\left\{x_{0}\right\} .
$$

We can scale problem (4.1) in $r^{-1} \Omega$ and suppose that $r=1$. Henceforth, we will denote by $B(z)$ a ball of radius 1 and center $z$. Given a function $u \in W_{0}^{s, p}(\Omega)$ and a ball $B(z) \subseteq \Omega^{c}$ such that $\partial B(z) \cap \partial \Omega=\left\{x_{0}\right\}$, the Kelvin transform of $u$ through $B(z)$ is given by

$$
u^{*}(x)=\frac{1}{|x-z|^{N-2 s}} u\left(z+\frac{x-z}{|x-z|^{2}}\right) .
$$

The map $\mathcal{I}_{z}(x):=z+(x-z) /|x-z|^{2}$ brings $\Omega$ to $\Omega_{z}:=\mathcal{I}_{z}(\Omega) \subseteq B(z)$. Furthermore, the function $u^{*} \in W_{0}^{s, p}\left(\Omega_{z}\right)$ satisfies

$$
(-\Delta)^{s} u^{*}(x)=\frac{1}{|x-z|^{N+2 s}}(-\Delta)^{s} u\left(\mathcal{I}_{z}(x)\right) .
$$

Given an externally tangent ball $B(z)$, the Kelvin transform thus brings any solution $u$ of (4.1) to a solution $v:=u^{*}$ in $\Omega_{z} \subseteq B(z)$ of

$$
\begin{cases}(-\Delta)^{s} v=g(x, v) & \text { in } \Omega_{z}, \\ v=0 & \text { in } \Omega_{z}^{c},\end{cases}
$$


where $z \notin \overline{\Omega_{z}}$ and the nonlinearity

$$
g(x, t):=g(|x-z|, t)=\frac{f\left(|x-z|^{N-2 s} t\right)}{|x-z|^{N+2 s}}
$$

satisfies the following properties:

(1) $g$ is Caratheodory, locally Lipschitz in the second variable and $C^{1}$ in the first;

(2) $g(r, t)$ is non-increasing in the first variable.

From any $x_{0} \in \partial \Omega$, we will now construct the exterior tangent ball $B(z), z=z\left(x_{0}\right)$ as above, and apply the moving plane method to $\Omega_{z}$. Without loss of generality we now suppose $z=0$, calling $\Omega_{0}=\widetilde{\Omega}$. Let $\nu_{x_{0}}$ be the interior normal to $B(z)$ at $x_{0}=\partial \widetilde{\Omega} \cap \partial B$ and observe that

$$
\inf _{\widetilde{\Omega}} x \cdot \nu_{x_{0}}=x_{0} \cdot \nu=-1,
$$

due to our normalization. For any $\lambda>-1$ we let

$$
\widetilde{\Omega}_{\lambda}^{\nu}=\widetilde{\Omega} \cap\{x \cdot \nu<\lambda\}, \quad x_{\lambda}^{\nu}=R_{\lambda}^{\nu}(x):=x+2(\lambda-x \cdot \nu) \nu,
$$

the latter being the reflection of $x$ through the hyperplane $\{x \cdot \nu=\lambda\}$. By the regularity of $\partial \widetilde{\Omega}$, there is $\varepsilon>0$ such that for all $\lambda \in]-1,-1+\varepsilon], R_{\lambda}^{\nu}\left(\widetilde{\Omega}_{\lambda}^{\nu}\right) \subseteq \widetilde{\Omega}$ (cf. [10, Theorem 5.7]). Moreover, by compactness, this $\varepsilon>0$ can be chosen independently of the particular $x_{0}$ from which the construction started. Moreover, if $\varepsilon<1$, it holds

$$
\left.\left.\left|x_{\lambda}^{\nu}\right| \leq|x| \quad \text { for any } \lambda \in\right]-1,-1+\varepsilon\right], x \in \widetilde{\Omega}_{\lambda}^{\nu} .
$$

Therefore, being $r \mapsto g(r, t)$ non-increasing for any $t \geq 0$,

$$
\left.\left.g(x, t) \leq g\left(x_{\lambda}^{\nu}, t\right), \quad \text { for any } \lambda \in\right]-1,-1+\varepsilon\right], x \in \widetilde{\Omega}_{\lambda}^{\nu}, t \geq 0 .
$$

The moving plane method as per [2, Proposition 4.4] can be applied, giving that

$$
s \mapsto v\left(x_{0}+s \nu\right) \quad \text { is increasing for } s \in[0, \varepsilon] .
$$

Recalling the definition of $v$ and $\nu=z-x_{0}$, we obtain

$$
s \mapsto \frac{1}{|1-s|^{N-2 s}} u\left(x_{0}-\nu \frac{s}{1-s}\right) \quad \text { is increasing for } s \in[0, \varepsilon],
$$

or, for $\xi=s /(1-s)$,

$$
\xi \mapsto|1+\xi|^{N-2 s} u\left(x_{0}-\xi \nu\right) \quad \text { is increasing for } \xi \in\left[0, \frac{\varepsilon}{1-\varepsilon}\right] .
$$

Since $\Omega$ is $C^{1,1}$, there is $\left.\bar{d} \in\right] 0,1[\operatorname{such}$ that $\Pi(x)=\operatorname{Argmin}\{|x-y|: y \in \partial \Omega\}$ is well defined and $C^{1,1}$. We define $d$ with $2 d<\min \{\bar{d}, \varepsilon\}<\varepsilon /(1-\varepsilon)$ so that the previous monotonicity gives

$$
\xi_{1} \leq d<\xi_{2} \leq 2 d \Rightarrow u\left(x_{0}-\xi_{1} \nu\right) \leq \frac{\left|1+\xi_{2}\right|^{N-2 s}}{\left|1+\xi_{1}\right|^{N-2 s}} u\left(x_{0}-\xi_{2} \nu\right) \leq|1+2 d|^{N-2 s} u\left(x_{0}-\xi_{2} \nu\right),
$$

concluding the proof.

Proof of Theorem 1.4. Rescaling the problem if necessary we can reduce to $\Omega=B_{r_{2} / r_{1}} \backslash B_{1}$. Let $R=r_{2} / r_{1}$ and let, for $\varepsilon>0$ sufficiently small,

$$
\bar{u}_{\varepsilon}=r_{1}^{\frac{2 s}{2^{*}-2-\varepsilon}} u_{\varepsilon}\left(r_{1} x\right)
$$



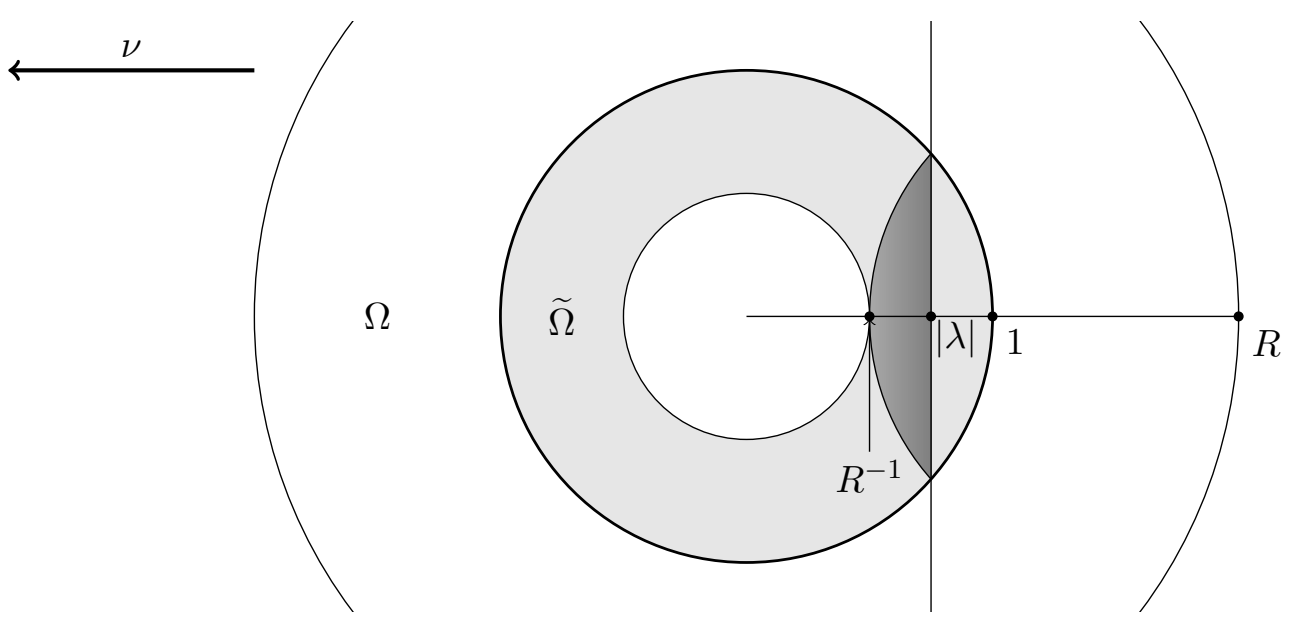

FiguRE 1. The optimal cap after inversion through the inner sphere

be the nonnegative solution to

$$
\begin{cases}(-\Delta)^{s} \bar{u}_{\varepsilon}=\bar{u}_{\varepsilon}^{2^{*}-1-\varepsilon} & \text { in } \Omega, \\ \bar{u}_{\varepsilon}=0 & \text { in } \Omega^{c} .\end{cases}
$$

Using the minimizing property (2.4) and scaling, it is readily checked that $\bar{u}_{\varepsilon}$ is a ground state solution of the previous problem, for any $\varepsilon>0$. We proceed by contradiction and suppose that $\left|\bar{u}_{\varepsilon}\right|^{2^{*}} \rightarrow \mathbb{S}^{\frac{N}{2 s}} \delta_{x_{0}}$ for some $1<\left|x_{0}\right|<2 /\left(1+R^{-1}\right)$, letting $M=2 /\left(1+R^{-1}\right)$. We omit for the time being the dependance on $\varepsilon$ and call $\bar{u}_{\varepsilon}=u$. The ball $B_{1}$ is externally tangent to $\Omega$ and we can perform the Kelvin transform through $B_{1}$, obtaining a solution to

$$
\begin{cases}(-\Delta)^{s} v=\frac{v^{2^{*}-1-\varepsilon}}{|x|^{\varepsilon(N-2 s)}} & \text { in } \mathcal{I} \Omega, \\ v=0 & \text { in } \mathcal{I} \Omega^{c} .\end{cases}
$$

Notice that $\mathcal{I} \Omega=B_{1} \backslash B_{1 / R}=: \widetilde{\Omega}$. Elementary geometric considerations (see figure 1 ) show that given any unit vector $\nu, a(\nu)=-1$ and

$$
\frac{1}{R}+2(1-|\lambda|)<1 \Rightarrow R_{\lambda}^{\nu}\left(\widetilde{\Omega}_{\lambda}^{\nu}\right) \subseteq \widetilde{\Omega}
$$

The previous condition actually gives the so called "optimal cap" (which in this case coincides with the maximal one), i.e.

$$
R_{\lambda}^{\nu}\left(\widetilde{\Omega}_{\lambda}^{\nu}\right) \subseteq \widetilde{\Omega} \quad \Leftrightarrow \quad-1<\lambda<-\frac{1+R^{-1}}{2}=-M^{-1}
$$

and the moving planes method ensures that

$$
s \mapsto v((s-1) \nu) \quad \text { is increasing for } 0 \leq s \leq 1-M^{-1} .
$$

In terms of $u$ we therefore have, for any $x_{0}$ such that $\left|x_{0}\right|=1$ and for $\nu=-x_{0}, t=1 /(1-s)$,

$$
t \mapsto t^{N-2 s} u\left(t x_{0}\right) \quad \text { is increasing for } 1 \leq t \leq M .
$$

Now if $|\bar{x}|<M-2 \theta$ with $\theta>0$, the previous monotonicty ensures that

$$
u_{\varepsilon}\left(t x_{0}\right)<\frac{r^{N-2 s}}{t^{N-2 s}} u_{\varepsilon}\left(r x_{0}\right) \leq M^{N-2 s} u_{\varepsilon}\left(r x_{0}\right), \quad \forall 1 \leq t \leq M-\theta \leq r \leq M,
$$




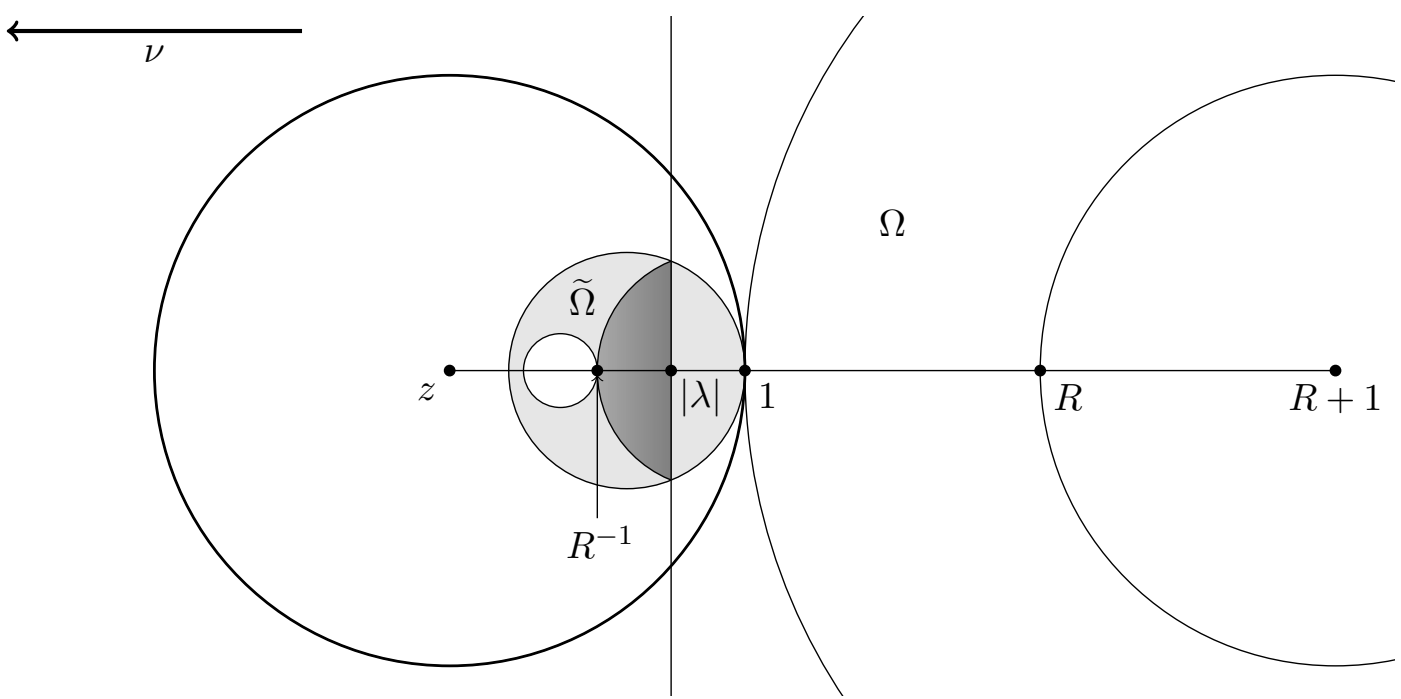

FiguRE 2. The optimal cap after inversion through the outer sphere

and thus

$$
\mathbb{S}^{\frac{N}{2 s}}=\lim _{\varepsilon \downarrow 0} \int_{B_{M-\theta} \backslash B_{1}} u_{\varepsilon}^{2^{*}} d x \leq C_{\theta} M^{N+2 s} \lim _{\varepsilon \downarrow 0} \int_{B_{M} \backslash B_{M-\theta}} u_{\varepsilon}^{2^{*}} d x=0,
$$

giving a contradiction. Similarly one can proceed if $|\bar{x}|>M+2 \theta$ : using this time Kelvin transform through an exterior unit ball of radius one (see figure 2), we obtain for any unit vector $x_{0}$

$$
t \mapsto(t+1)^{N-2 s} u\left((R-t) x_{0}\right) \quad \text { is increasing for } 0 \leq t \leq M,
$$

and integrating $u_{\varepsilon}^{2^{*}}$ over $B_{R} \backslash B_{M+\theta}$ and $B_{M+\theta} \backslash B_{M}$ gives the desired contradiction.

\section{REFERENCES}

[1] F. Atkinson, L. Peletier, Elliptic equations with nearly critical growth, J. Differential Equations 70 (1986), 349-365. 2

[2] B. Barrios, L. Montoro, B. Sciunzi, On the moving plane method for nonlocal problems in bounded domains, J. Anal. Math. to appear http://arxiv.org/abs/1405.5402. 3, 15

[3] L. Brasco, G. Franzina, Convexity properties of Dirichlet integrals and Picone-type inequalities, Kodai Math. J. 37 (2014), 769-799. 6

[4] L. Brasco, S. Mosconi, M. Squassina, Optimal decay of extremals for the fractional Sobolev inequality, Calc. Var. PDE, to appear. http://arxiv.org/abs/1508.00132. 10

[5] L. Brasco, E. Parini The second eigenvalue of the fractional p-Laplacian, Adv. Calc. Var., to appear. http://dx.doi.org/10.1515/acv-2015-0007.1, 6

[6] L. Brasco, M. Squassina, Y. YAnG, Global compactness results for nonlocal problems and applications, preprint. 2, 5

[7] H. Brezis, L. Peletier, Asymptotic for elliptic equations involving critical growth, Progress in Nonlinear Differential Equations and Applications 1, Birkhäuser Boston, Inc., Boston, 1989, 149-192. 2

[8] M. Flucher, Variational Problems with Concentration, Progress in Nonlinear Differential Equations and Applications 36, Birkhäuser Boston, Inc., Boston, 1999. 2

[9] M. Flucher, A. Garroni, S. Müller, Concentration of low energy extremals: Identification of concentration points, Calc. Var. PDE 14 (2002), 483-516. 2

[10] L.E. Fraenkel, An introduction to maximum principles and symmetry in elliptic problems, Cambridge Tracts in Mathematics 128, Cambridge University Press, Cambridge, 2000. 15

[11] J. Garcia Azorero, I. Peral Alonso, On limits of solutions of elliptic problems with nearly critical exponent, Commun. Partial Differential Equations 17 (1992), 2113-2126. 2, 11 
[12] Z.-C. HaN, Asymptotic approach to singular solutions for nonlinear elliptic equations involving critical Sobolev exponent, Annales de l'Institut Henri Poincaré (C) Analyse Non Linéaire 8 (1991), 159-174. 2

[13] A. Iannizzotto, S. Mosconi, M. Squassina, $H^{s}$ versus $C^{0}$-weighted minimizers, NoDEA Nonlinear Differential Equations Applications, 22 (2015), 477-497. 6

[14] A. Innnizzotto, S. Mosconi, M. Squassina, Global Hölder regularity for the fractional $p$-Laplacian, Rev. Mat. Iberoam. to appear. http://arxiv.org/abs/1411.2956. 1, 6

[15] S. JAfFARD, Analysis of the lack of compactness in the critical Sobolev embeddings, J. Funct. Anal. 161 (1999), 384-396. 2

[16] T. Kuusi, G. Mingione, Y. Sire, Nonlocal equations with measure data, Comm. Math. Phys. 337 (2015), 1317-1368. 1

[17] P.L. Lions, The concentration-compactness principle in the calculus of variations. The limit case, Part I, Rev. Mat. Iberoam. 1 (1985), 145-201. 7, 8

[18] A. Musesti, M. Squassina, Asymptotics of solutions for fully nonlinear elliptic problems at nearly critical growth, Z. Anal. Anwend. 21 (2002), 185-201. 2

[19] S. Mosconi, K. Perera, M. Squassina, Y. Yang, The Brezis-Nirenberg problem for the fractional p-Laplacian, preprint http://arxiv.org/abs/1508.00700. 3, 10

[20] G. Palatucci, p-Laplacian problems with critical Sobolev exponent, Asymptotic Anal. 73 (2011), 37-52. 2

[21] G. Palatucci, A. Pisante, Improved Sobolev embeddings, profile decomposition, and concentrationcompactness for fractional Sobolev spaces, Calc. Var. PDE 50 (2014), 799-829. 2

[22] G. Palatucci, A. Pisante, Y. Sire, Subcritical approximation of a Yamabe type non local equation: a Г-convergence approach, Ann. Scuola Norm. Sup. Pisa Cl. Sci. 14 (2015), 819-840. 2

[23] O. REY, Proof of the conjecture of H. Brezis and L. A. Peletier, Manuscripta Math. 65 (1989), 19-37. 2

[24] M. Willem, Minimax theorems, Progress in Nonlinear Differential Equations and their Applications 24, Birkhäuser Boston, Inc., Boston, 1996. 5

(S. Mosconi) Dipartimento di Informatica

UNIVERSITÀ DEGLI STUdi DI VERONA

CÁ Vignal 2, Strada Le Grazie 15, 37134 Verona, Italy

E-mail address: sunra.mosconi@univr.it

(M. Squassina) Dipartimento di Informatica

UNIVERSiTÀ DEGLi STUdi Di VERONA

Cá Vignal 2, Strada le Grazie 15, 37134 Verona, Italy

E-mail address: marco.squassina@univr.it 